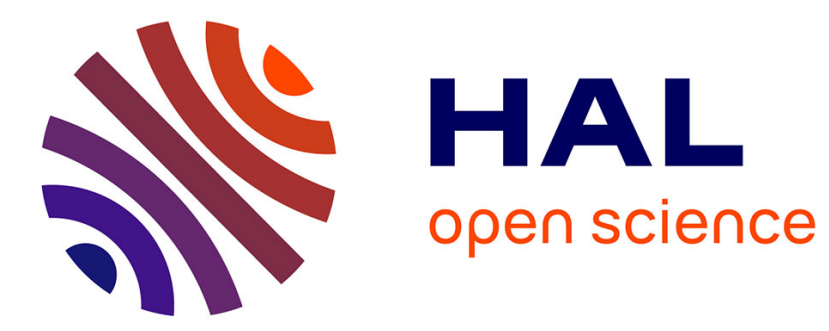

\title{
Resonant electron emission of silver spheroids induced by laser surface plasmon excitation
}

P Monchicourt, Michèle Raynaud, H Saringar, J. Kupersztych

\section{To cite this version:}

P Monchicourt, Michèle Raynaud, H Saringar, J. Kupersztych. Resonant electron emission of silver spheroids induced by laser surface plasmon excitation. Journal of Physics: Condensed Matter, 1997, 9, pp.5765 - 5775. 10.1088/0953-8984/9/27/008 . hal-01949723

\section{HAL Id: hal-01949723 \\ https://hal.science/hal-01949723}

Submitted on 26 Dec 2020

HAL is a multi-disciplinary open access archive for the deposit and dissemination of scientific research documents, whether they are published or not. The documents may come from teaching and research institutions in France or abroad, or from public or private research centers.
L'archive ouverte pluridisciplinaire HAL, est destinée au dépôt et à la diffusion de documents scientifiques de niveau recherche, publiés ou non, émanant des établissements d'enseignement et de recherche français ou étrangers, des laboratoires publics ou privés. 


\title{
Resonant electron emission of silver spheroids induced by laser surface plasmon excitation
}

\author{
P Monchicourt, M Raynaud, H Saringar and J Kupersztych \\ Commissariat à l'Energie Atomique, Service de Recherche sur les Surfaces et l'Irradiation de la \\ Matière, Centre d'Etudes de Saclay, 91191 Gif sur Yvette Cédex, France \\ Received 25 October 1996, in final form 2 April 1997
}

\begin{abstract}
Laser excitation of surface plasmons on a silver granular surface has been studied as a function of light frequency. Both light absorption and electron emission have been measured by varying continuously the wavelength between 275 and $700 \mathrm{~nm}$. Resonance effects at the Fröhlich frequencies of the silver spheroids are displayed and a correlation between absorption and emission is clearly exhibited. The lux-ampere characteristics performed at selected increasing wavelengths display slopes with integer orders, indicating that the emission process remains a photoeffect even in the presence of a surface-plasmon resonance. The photoemission process shows net switches of these slopes that turn from linear into quadratic and then cubic integer values. The position of the first switch yields $W_{s}=4.15 \pm 0.05 \mathrm{eV}$ for the work function of the granular surface. The position of the second switch defines the threshold energy for the two-photon emission process, which is found to differ significantly from the expected photon minimum energy $\hbar \omega=\frac{1}{2} W_{s}$.
\end{abstract}

\section{Introduction}

Absorption of light by metallic surfaces can be greatly enhanced when collective modes of the electron gas are excited at the boundary of the metal (Forstman and Gerhardts 1986). These modes - called surface plasmons (SP) — are high-frequency plasma oscillations which can be sustained by the electrons near the metal-vacuum interface. Resonant excitation of SPs can be achieved by an electromagnetic wave at frequencies which match the normal mode frequencies of the system and are characteristic of the nature of the metal and the geometry of the surface. Beyond the fundamental importance of studying these collective excitations, the question of the relaxation of SP resonances is of major interest since this relaxation acts both as a limiting process for the amplitude of the resonances and as an additional energy input for the electron population. This input energy is then redistributed through various channels (electron-electron collisions, single-particle excitation, interband transitions, emission of light, etc) and in various components (electron gas, lattice vibrations, hot-electron generation (Tsang et al 1990), etc). A good understanding of this redistribution step is strongly needed in order to control the coupling between light and metallic surfaces. However, the relative efficiencies and relaxation rates of these various redistribution channels involve very short time scales which are only accessible with subpicosecond experiments (Fann et al 1992, Steinmuller-Nethl et al 1992, Bigot et al 1995). Therefore, the measurements are performed in most cases not during but after the redistribution step by analysing the resulting effect over one component of the metallic system.

A particularly interesting effect of this energy redistribution is electron emission from the surface. Besides the well known photoelectric and thermoionic effects, electron emission 
is observed to be enhanced when the SPs are excited (Macek et al 1972, Rudolf and Steinmann 1977, Tsang et al 1990) with an even greater efficiency for rough or granular surfaces (Stuckless and Moskovits 1989, Sabary et al 1991). This emission seems well suited to studying experimentally both the collective behaviour of a metallic surface and the processes which control the relaxation of energy. However, such experimental observations have been generally made at discrete or in restricted ranges of frequencies, and variations in this emission in a large spectral range (i.e. from UV to IR) are at present not available. In the present work, we shall show, by varying continuously the light frequency in this domain, that absorption and electron emission are intimately correlated and display a resonant behaviour at the SP frequencies.

SP excitation by light depends obviously on the surface geometry. For a smooth plane surface, we are dealing with a continuous spectrum of SP modes $\omega(k)$ propagating along the surface. However, these propagating modes cannot be directly excited by light at a vacuum interface because the phase velocity of the SP is slower than that of a photon of the same energy. Thus, conservation of momentum cannot be satisfied, except by using a glass prism, such as in the ATR method (Otto 1968, Kretschmann 1971), or through roughness or periodicity of the surface (Raether 1988). For spherical geometry, the normal modes are oscillation modes, also termed 'localized' plasmons (Raether 1988). This geometry is of particular interest since the SP mode spectrum of such systems has discrete frequency values, the first of which is the Fröhlich frequency (Bohren and Huffman 1983). Thus, a single mode can be excited by selecting the polarization of light and sweeping its frequency. The second advantage of using spherical systems is that absorption of light is maximized because of the localized nature of the SP modes. Thus, intense electron emission is achieved, which makes granular films very attractive as photocathodes or pulsed electron sources (Dudek and Sabary 1990, Sabary and Dudek 1990). Hence, we have used this geometry in the present work by resonantly driving such surface (Fröhlich) modes on metallic spheroids with a tunable laser. Resonance effects have been obtained by tuning the wavelength between 275 and $700 \mathrm{~nm}$, both in the absorption of radiation and in electron emission from the surface. The continuous variation in the wavelength demonstrates that electron emission spectra are intimately correlated with radiation absorption (section 3). In order to get quantitative informations about the electron emission process, an experimental determination of the work function has been performed (section 4). Lux-ampere characteristics (electron current versus laser intensity) at selected laser frequencies are analysed in section 5 . These characteristics exhibit slopes with integer values switching from order 1 to 2 and 2 to 3 as the wavelength is increased. The position of the two switchings yields new information about the photoemission process, which is discussed in section 6 .

\section{Experimental arrangement}

The present experiment has been performed on Ag spheroids deposited on a $\mathrm{Al}$ oxidized substrate prepared and characterized as described elsewhere (Dudek and Sabary 1990, Sabary and Dudek 1990). Two groups of Ag particles with different shapes are produced: nearly spherical particles and prolate ellipsoids with major axis $a$ parallel to the substrate and minor axis $b$ perpendicular to the surface (Dudek and Sabary 1990, Sabary and Dudek 1990). It has to be emphasized that, for spherical particles, the standard experimental frequency of the Fröhlich mode in silver corresponds to an energy of $3.6 \mathrm{eV}$ (Skillman and Berry 1968). For prolate ellipsoids, the Fröhlich mode splits into two modes with frequencies $\omega_{\|}$and $\omega_{\perp}$ depending on the form factor $b / a$ (Skillman and Berry 1968). The highest frequency $\omega_{\perp}$ corresponds to the minor axis $b$, and the lowest frequency $\omega_{\|}$to the major 
axis $a$. For the $\mathrm{Ag}$ granular film under investigation, the number density of $\mathrm{Ag}$ spheroids is about $10^{10}$ per square centimetre of the substrate area and the spheroid size is about 10-50 nm (Dudek and Sabary 1990, Sabary and Dudek 1990). The Ag granular film is held vertically, with translation and rotation adjustments, at the centre of an ion-pumped vacuum chamber $\left(10^{-8}\right.$ mbar or less) closed by silica windows (see figure 1). The irradiation light comes from a nanosecond Nd:YAG pumped tunable dye laser (Quantel TDL 50 system) which produces monochromatic light pulses of $8 \mathrm{~ns}$ FWHM, $10 \mathrm{~Hz}$ in repetition rate, and a wavelength tunable in the range $275-700 \mathrm{~nm}$ with a linewidth of a few hundredths of a nanometre. This tunable photon energy $(\hbar \omega=4.5-1.8 \mathrm{eV})$ is obtained in one of three ways:

(i) directly from the dye or

(ii) after frequency doubling or

(iii) after mixing with the fundamental frequency of the YAG pump.

Six different dyes were necessary to obtain the required range of wavelengths. The intensity and polarization of the incident light can be adjusted with a half-wave plate + Glan-Taylor prism system. The incidence angle can be varied by rotating the Ag target around a vertical axis. The optical axis and the beam cross section are imposed by a circular diaphragm $1 \mathrm{~mm}$ in diameter. The target is irradiated by the beam without any focusing. The incident and reflected light intensities are measured for each laser pulse by two fast photodiodes calibrated with an energy meter. The electrons emitted from the surface are collected through a plane grid on an electron multiplier. After amplification, the repetitive pulse of current is monitored, together with the two photodiode pulses, on a digital oscilloscope LeCroy 9354A. The three pulses are recorded as single-shot data when the peak intensity of incident light matches a preselected value. The results presented below correspond to peak values of both laser intensity and electron current pulses. Two sets of data have been obtained in this work:

(i) variation in light reflectivity and electron emission versus $\lambda$ for a constant laser intensity;

(ii) 'lux-ampere' characteristics for selected $\lambda$ displaying variation in electron emission versus light intensity.

These data enable us to analyse selectively the photoemission process within and without SP resonance excitation.

\section{Excitation of surface plasmon resonances}

The SP resonances are first characterized by varying continuously the laser wavelength $\lambda$ and keeping the laser intensity fixed $\left(E_{L} \simeq 2 \times 10^{4} \mathrm{~W} \mathrm{~cm}^{-2}\right)$. The signature of SP resonances is obtained by selective excitation of the previously defined $\omega_{\|}$and $\omega_{\perp}$ modes. This is achieved by using p- or s-polarized light; the behaviour of reflectivity versus $\lambda$ is shown in figure 2 at a light incidence angle of $62^{\circ}$, which optimizes the signal amplitude for both polarizations.

Reflectivity of the Ag granular surface exhibits three minima at $\lambda \simeq 335 \mathrm{~nm}, 375 \mathrm{~nm}$ and $510 \mathrm{~nm}$ for p-polarized light. For s polarization, the $335 \mathrm{~nm}$ feature is lacking and the second and third resonances remain at nearly the same wavelengths. These observations are in agreement with those made by Dudek and Sabary (1990) and Sabary and Dudek (1990). They have been interpreted as being due to the existence of two groups of particles on the surface, with average form factors $b / a \simeq 0.9$ and 0.3 . The corresponding resonance 


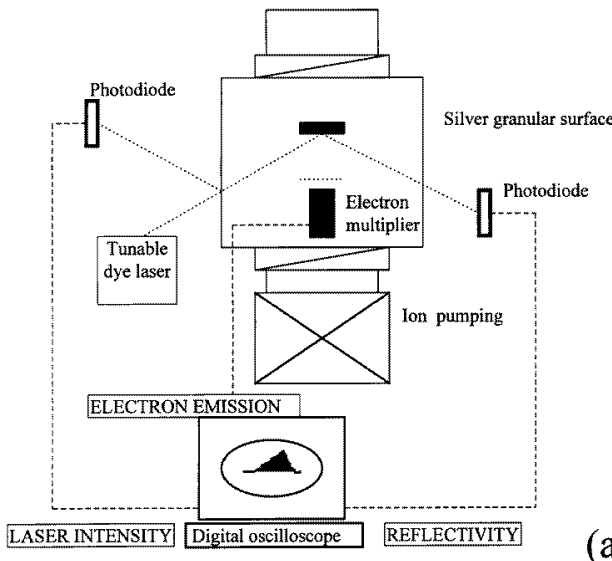

(a)

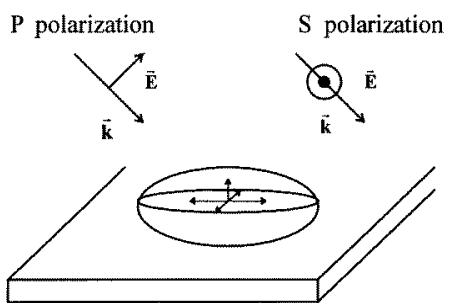

(b)

Figure 1. (a) Schematic view of the experimental set-up. (b) Definition of $\mathrm{p}$ and s polarizations for the granular film. The Ag particles are of two sorts: spheres, and prolate ellipsoids with major axis $a$ parallel to the surface, and minor axis $b$ perpendicular. For s polarization, the electric field is parallel to the surface and drives only the modes along the major axis. For $\mathrm{p}$ polarization, the field drives components along both $a$ and $b$.

energies are $\hbar \omega_{\perp} \simeq 3.7 \mathrm{eV}$ and $\hbar \omega_{\|} \simeq 3.3 \mathrm{eV}$ and $2.4 \mathrm{eV}$, respectively. For s-polarized light, the electric field is parallel to the surface of the substrate and excites only the $\omega_{\|}$ modes. In the case of p-polarized light, the electric field has components in both directions and drives simultaneously the $\omega_{\|}$and $\omega_{\perp}$ modes (see figure 1 for the definition of the light polarization for granular films). If we assume that the sum of reflectivity and absorption coefficients is unity, absorption is found to reach about $70-90 \%$ for p-polarized light and 40-50\% for s-polarized light at maximum resonance of the three modes. It can be also noticed that absorption is increased in $\mathrm{p}$ polarization by a factor of about 2 .

Turning now to electron emission associated with this efficient absorption, the two first SP resonances are seen to be associated with two resonances in electron emission at the same wavelengths. This observed matching in energy demonstrates that electron emission closely follows the absorption variation versus $\lambda$. This suggests that this emission represents a nonnegligible channel for SP relaxation at these two wavelengths compared with other relaxation mechanisms, such as thermal redistribution or light emission. An obviously different regime is observed in the case of the third resonance at $510 \mathrm{~nm}$ since electron emission does not follow the absorption variations; in figure 2, the former is seen to decrease by two orders of magnitude with increasing wavelength from 430 to $550 \mathrm{~nm}$ whereas the latter remains at around $20-40 \%$. This feature is analysed in more detail in section 5. As displayed in figure 2, knowledge of both light absorption and electron emission as functions of $\lambda$ seems essential because, first, very important variations in the electron signal occur in restricted wavelength ranges and, second, a close correlation does not systematically hold over the whole spectrum.

As is also shown in figure 2, electron emission is detected for photon energies below the threshold of the photoelectric effect, which is expected to be $4.3 \mathrm{eV}$ for a polycrystalline clean Ag plane surface (Ashcroft and Mermin 1987). As our sample was made of granular silver and might have suffered surface contamination, its work function might differ from the standard value of $4.3 \mathrm{eV}$. Therefore, a crucial test was to determine experimentally the work function of the Ag granular surface under investigation. 


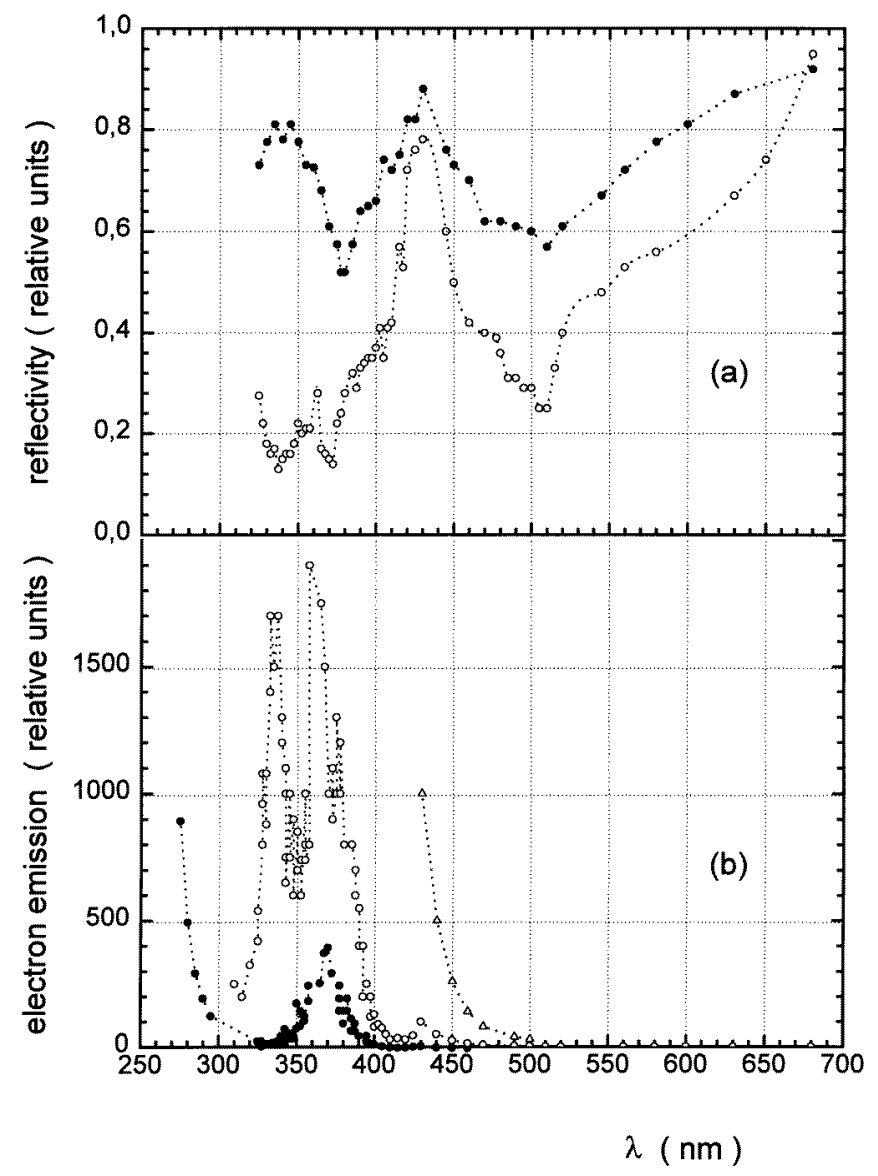

Figure 2. (a) Reflectivity and (b) electron emission of the granular surface as a function of wavelength for p-polarized $(\mathrm{O})$ and s-polarized $(\bullet)$ light. The data points are connected for clarity. The statistical uncertainty is about $5 \%$ for (a) and $10 \%$ for (b). Absolute values of reflectivity correspond, to within $10-15 \%$, to the vertical scale of (a). The uncertainty comes from the transmission of the silica windows and from the reflectivity of the Al substrate. For (b), the current density, in amperes per square centimetre, can be obtained (within a factor of 3 ) by multiplying the vertical scale by $2 \times 10^{-11}$. The open triangles correspond to the p signal magnified by a factor of 10 and display an exponential decay from 430 to $500 \mathrm{~nm}$.

\section{Experimental determination of the work function}

The expected threshold for the photoelectric effect $(4.3 \mathrm{eV})$ corresponds to a photon wavelength $\lambda \simeq 288 \mathrm{~nm}$. Above this threshold $(\lambda<288 \mathrm{~nm})$, ejection of an electron from the surface by a photon is expected, from the Einstein law, to give a linear variation in the electron current $I_{e}$ versus laser intensity $I_{L}$. Below the threshold, the normal linear photoemission is expected to be vanishingly small and is replaced by non-linear processes (e.g. a quadratic process). This behaviour is confirmed in figure 2 , where an abrupt decay of the emission versus $\lambda$ is observed from 280 to $310 \mathrm{~nm}$. This is consistent with the decay corresponding to the exponential tail of the Fermi distribution for the electron energy (Cardona and Ley 1978). Moreover, the $\log I_{e}$ versus $\log I_{L}$ plot (lux-ampere 
characteristics) reported on figure 3 confirms the switch from a slope of 1 below $280 \mathrm{~nm}$ to a slope of 2 above $320 \mathrm{~nm}$. The energy interval corresponding to these two wavelengths $(4.4 \mathrm{eV}>\hbar \omega>3.9 \mathrm{eV})$ is a direct (but approximate) indication of the work function $W_{s}$. A more accurate determination can be obtained from the electron emission variation law of a Fermi distribution near threshold, as given by the modified Fowler-Dubridge law (Fowler 1931, Cardona and Ley 1978): $I_{e} \propto\left(\hbar \omega-W_{s}\right)^{2}$ for $\mathrm{kT} \ll \hbar \omega-W_{s} \ll W_{s}$. To this aim, a plot of $I_{e}^{1 / 2}$ versus $\hbar \omega$ should yield straight lines which can be extrapolated to zero current in order to determine $W_{s}$. The result of this procedure is shown in figure 4 at four different laser intensities. The straight lines represent the best fits through the data obtained for $\hbar \omega>4.27 \mathrm{eV}$ at each intensity below $3.5 \times 10^{4} \mathrm{~W} \mathrm{~cm}^{-2}$, and for $\hbar \omega>4.35 \mathrm{eV}$ at $3.5 \times 10^{4} \mathrm{~W} \mathrm{~cm}^{-2}$. These lines verify the Fowler approximation $I_{e}^{1 / 2} \propto \hbar \omega-W_{s}$ and are consistent with a pure photoeffect, i.e. electron ejection after photon absorption without significant heating of the Fermi distribution. The intersection of the different lines with the $\hbar \omega$ axis occurs between 4.14 and $4.17 \mathrm{eV}$. This defines our experimental work function $W_{s}=4.15 \pm 0.05 \mathrm{eV}$. This value is in good agreement with standard published values (Ashcroft and Mermin 1987). It can be concluded that the effects of impurities and surface contamination are negligible in our sample and that electron ejection from the granular surface is not substantially affected by the small size or curved shape effects of the spheroids (cf discussion in section 5). The observed departures from straight lines for $\hbar \omega<4.35 \mathrm{eV}$ and for $I_{L}=3.5 \times 10^{4} \mathrm{~W} \mathrm{~cm}^{-2}$ in figure 4 is probably due to a significant heating of the electron gas $\left(\mathrm{kT} \geqslant \hbar \omega-W_{s}\right)$. In this case, emission of an electron from the tail of the Fermi distribution by a photon with energy below $W_{s}$ is made possible (Cardona and Ley 1978). As observed in figure 4, this heating effect (i.e. departure from straight lines) is obviously more efficient as $\hbar \omega$ is near threshold or $I_{L}$ (or kT) is high. The resulting error in $W_{s}$ is of the order of the thermal spreading $(0.1 \mathrm{eV}$ or less in the present case).

\section{Lux-ampere characteristics at surface plasmon resonances}

The quadratic photoeffect observed in section 4 above $W_{s}=4.15 \mathrm{eV}$ suggests a process involving the absorption of two photons. In fact, multiphoton processes have already been observed at high laser intensities (of the order of $1 \mathrm{GW} \mathrm{cm}^{-2}$ (Bechtel et al 1977)). However, such coherent processes seem very unlikely at the rather weak laser intensities achieved in the present experiment, i.e. tens to hundreds of kilowatts per square centimetre. Therefore, a two-photon process can be understood in our case as depicting a sequential absorption of two photons by the electron system. According to this scheme, a quadratic effect is expected for $2.07 \mathrm{eV}<\hbar \omega<4.15 \mathrm{eV}$, i.e. for $598 \mathrm{~nm}>\lambda>299 \mathrm{~nm}$. Below $2.07 \mathrm{eV}$ (above $598 \mathrm{~nm}$ ), the energy of two photons becomes insufficient and three photons are then needed for an electron to be ejected.

Accordingly, a $\log -\log$ plot of $I_{e}$ versus $I_{L}$ in this wavelength range exhibits in figure 5 a slope of 2 at 335 and $375 \mathrm{~nm}$, i.e. when the two first SP resonances are selectively excited. This is not true for the third resonance at $\lambda \simeq 510 \mathrm{~nm}$. In fact, the slope remains of second order from 299 to $480 \mathrm{~nm}$ and is observed in figure 6 to switch from order 2 to order 3 between 480 and $510 \mathrm{~nm}$, i.e. around $2.4 \mathrm{eV}$. This switching is associated with a strong decay of the electron emission; as seen in figure 6 for $I_{L}=10^{5} \mathrm{~W} \mathrm{~cm}^{-2}$, there is a decrease of three orders of magnitude between the signal amplitudes at 480 and $550 \mathrm{~nm}$. The occurrence of such a switch at around $510 \mathrm{~nm}$ explains why electron emission does not follow the absorption variation versus wavelength, as already reported in figure 2 (see section 3). Above this wavelength, the slope remains at 3 up to $700 \mathrm{~nm}$, which is the highest 


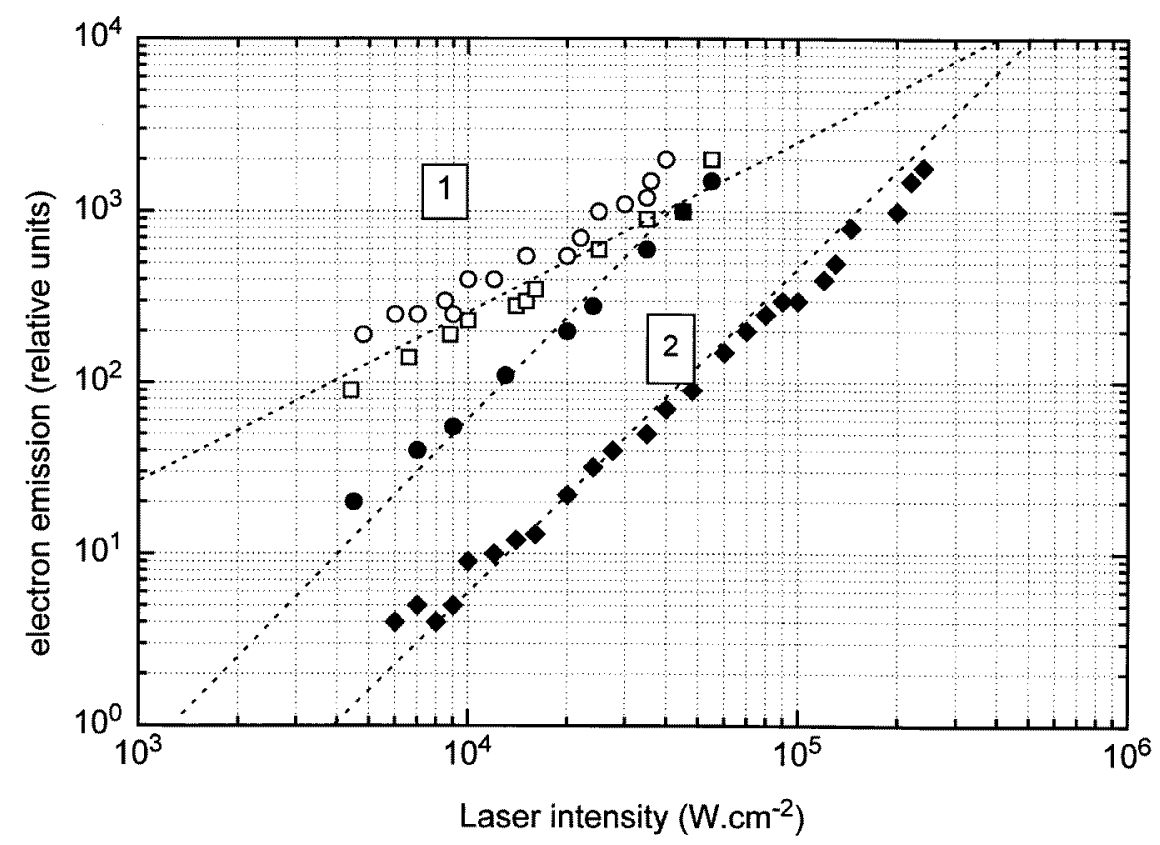

Figure 3. Lux-ampere characteristics of the electron signal for four different laser wavelengths: $\bigcirc, 275 \mathrm{~nm} ; \square, 280 \mathrm{~nm} ; \bullet, 310 \mathrm{~nm} ;, 320 \mathrm{~nm}$. The broken straight lines show slopes of 1 and 2 as guides to the eye. An abrupt change in slope from 1 to 2 occurs when the threshold for the one-photon photoelectric effect is crossed, i.e. between 280 and $310 \mathrm{~nm}$. Above $320 \mathrm{~nm}$, the order remains 2 until $480 \mathrm{~nm}$.

accessible wavelength. This slope is observed to change by integer values, even at the SP resonance. This important feature implies that the process of electron ejection remains a photoeffect (two-step or three-step processes in that range of $\lambda$ ) even when the SP resonance is excited. Moreover, a constant slope of the electron emission versus laser intensity over three orders of magnitude for each selected wavelength confirms the absence of heating of the electrons (e.g. 'thermally assisted emission' depicted by Yen et al (1980)). Above $510 \mathrm{~nm}$ $(\hbar \omega=2.45 \mathrm{eV})$, three photons become necessary for an electron to be ejected from the metal surface, whereas two photons are sufficient below this value. This defines an energy threshold of $2.45 \mathrm{eV}$ for an electron to be ejected after two-photon absorption. According to this value, an energy threshold of $4.9 \mathrm{eV}$ (twice $2.45 \mathrm{eV}$ ) is expected for electron ejection at this photon energy. Indeed, this differs from the measured work function $W_{s}=4.15 \mathrm{eV}$. In fact, this quite unexpected result means that more energy is needed for the two-photon process than for the one-photon effect. The difference of $0.75 \mathrm{eV}$ is well outside our error bars and cannot be due to an experimental artefact. Thus, the question is raised of which processes are involved to induce such an energy difference.

(i) A degradation of the input energy between the two absorption events occurs. In this scheme, the subsequent heating of the electron gas would be observed as thermoionic emission. This would increase the slope of the lux-ampere characteristics (Logothetis and Hartman 1969, Anisimov et al 1978, Yen et al 1980). This is not observed, however.

(ii) The electron ejection process is modified, inasmuch as the work function is shifted towards higher energies in the presence of the SP. Many different processes for electron ejection have been proposed, depending on the coupling conditions between light and 


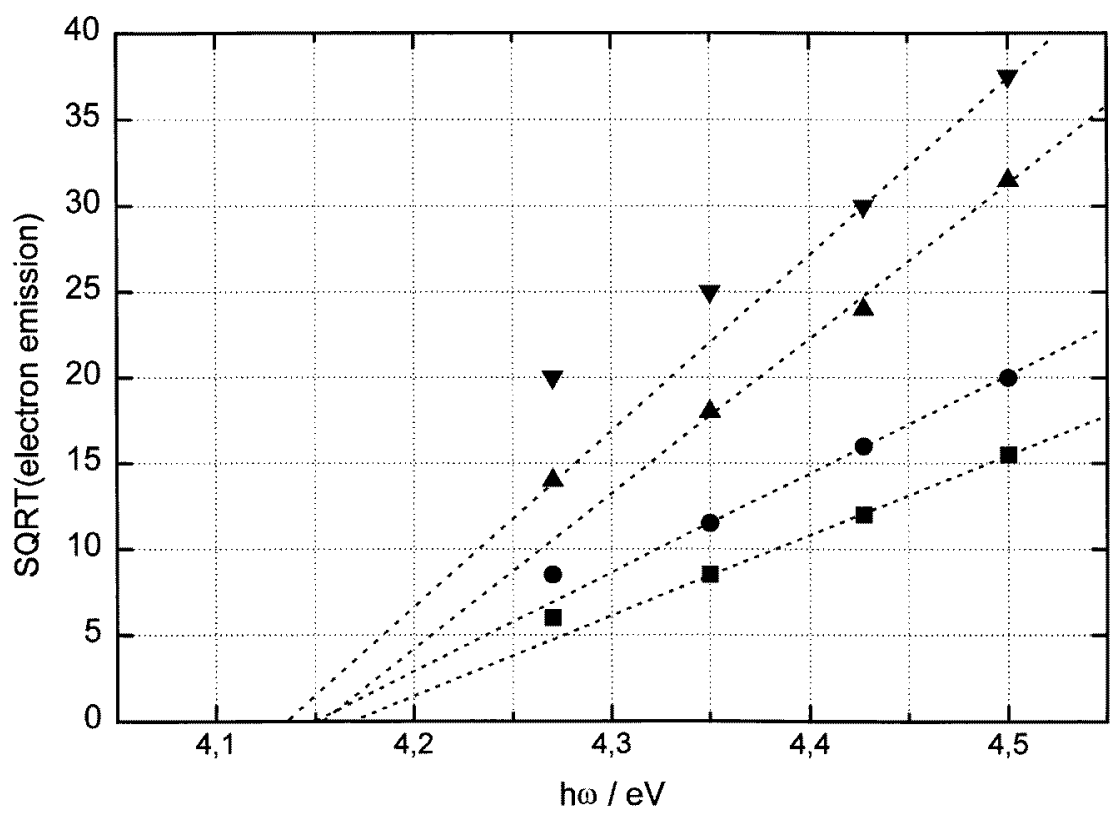

Figure 4. Modified Fowler plot of the electron signal $I_{e}$ versus $\hbar \omega$ measured near threshold for different laser intensities $I_{L} . \mathbf{\square}, 6 \times 10^{3} \mathrm{~W} \mathrm{~cm}^{-2} ; \bullet, 1.2 \times 10^{4} \mathrm{~W} \mathrm{~cm}^{-2} ; \boldsymbol{\Delta}, 2.5 \times 10^{4} \mathrm{~W} \mathrm{~cm}^{-2}$; $\mathbf{\nabla}, 3.5 \times 10^{4} \mathrm{~W} \mathrm{~cm}^{-2}$. The broken straight lines are the best fits obtained through the experimental points for $\hbar \omega>4.27 \mathrm{eV}$ and $I_{L}<3.5 \times 10^{4} \mathrm{~W} \mathrm{~cm}^{-2}$, and $\hbar \omega>4.35 \mathrm{eV}$ for $I_{L}=3.5 \times 10^{4} \mathrm{~W} \mathrm{~cm}^{-2}$. These lines verify the Fowler law near threshold: $I_{e}^{1 / 2} \propto \hbar \omega-W_{s}$. For smaller $\hbar \omega$ and higher $I_{L}$, departures are observed and are probably due to heating effects. The intersection points of the different straight lines with the $\hbar \omega$ axis defines the work function $W_{s}=4.15 \pm 0.05 \mathrm{eV}$.

electrons: an $n$-photon photoelectric effect or thermoionic effect in the case of high-intensity laser-metal irradiation (Logothetis and Hartman 1969, Anisimov et al 1978, Yen et al 1980), direct ejection of hundreds of electronvolt electrons out of the resonance region observed in laser-plasma experiments (Estabrook and Kruer 1978), evaporation of neutral atoms or molecules from laser-heated clusters (Hoheisel et al 1988) followed by post-ionization in the laser field, etc. Some of these effects are known to modify the work function of a metal surface. However, they cannot explain the large blue shift observed for two-photon emission together with the absence of shift for the one-photon emission. Geometrical effects or pollution of the surface cannot be invoked either since they would yield the same shift for both one- and two-photon emissions (Wood 1981, Lundqvist and March 1983). Also, thermal effects cannot be put forward for the same reason since the additional energy in the Fermi distribution would shift the threshold towards lower energies (Cardona and Ley 1978). Space charge effects might also be invoked for our spheroids deposited on an Al oxidized substrate which may behave as an insulator layer. In that case, the potential barrier would modify the emitted electron current independently of the wavelength of the light (Cardona and Ley 1978) and this is not observed.

Finally, an important indication drawn from the above observations deserves to be noted; the two-photon threshold is observed within the third SP resonance of the granular surface whereas the one-photon threshold occurs in an energy range far from any SP resonance. As we find again the standard value of $W_{s}$ out of any SP resonance, we may therefore conclude 


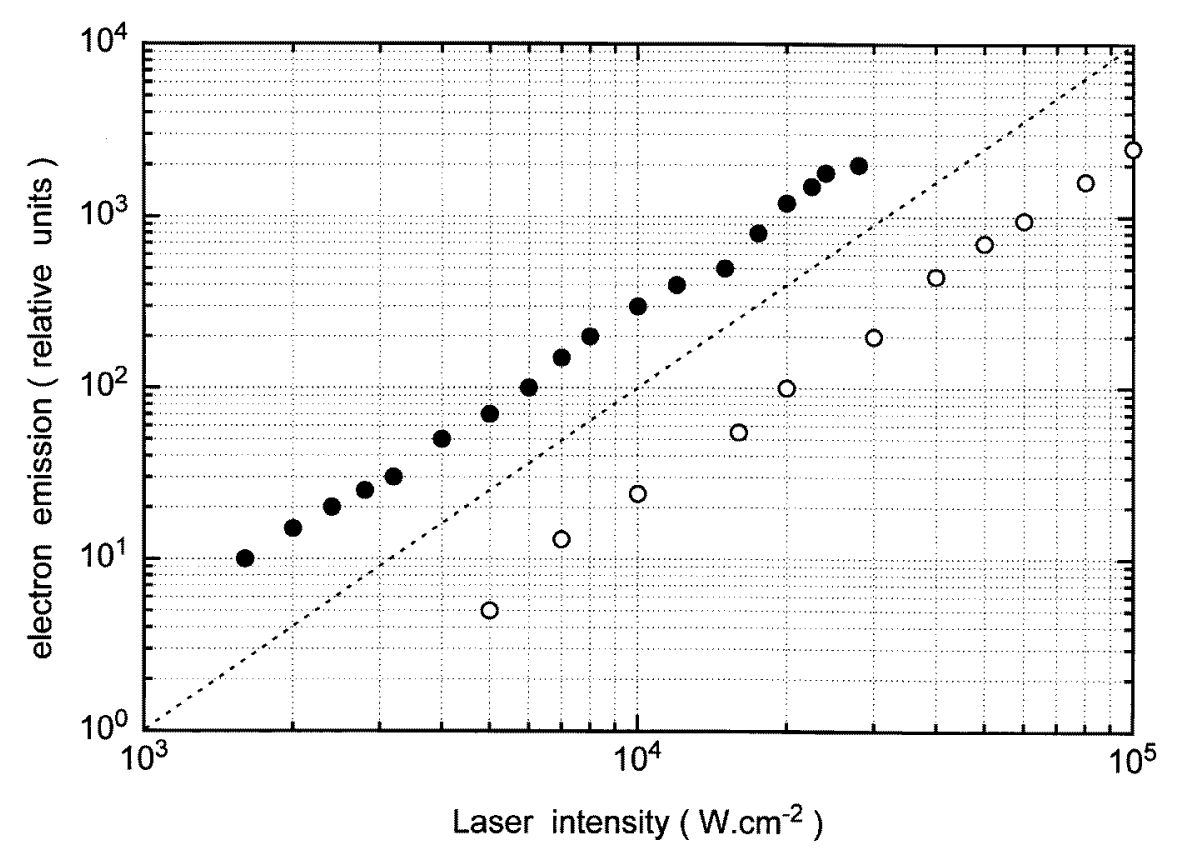

Figure 5. Lux-ampere characteristics of the electron signal measured for the two SP resonances at $335 \mathrm{~nm}(\bullet)$ and $375 \mathrm{~nm}(\mathrm{O})$. The broken straight line indicates a slope of 2.

that modifications of the electron ejection characteristics occur only in the presence of SP resonance.

\section{Conclusive remarks}

In this work, we have shown that light excitation of SPs on silver spheroids leads to resonance effects in both light absorption and electron emission. Important information can be drawn from these correlated observations. The lux-ampere characteristics display slopes with integer orders for the photo-effect; electron emission switches from a linear behaviour to quadratic and then cubic when the laser wavelength is tuned from 275 to $700 \mathrm{~nm}$. Owing to the rather low light intensities involved in our experiments $\left(10^{4}-10^{5} \mathrm{~W} \mathrm{~cm}^{-2}\right)$, these processes cannot involve coherent absorption events but, rather, sequential events.

From the observed electron emission switches, a new and important result is obtained, i.e. the observation of a modification of the potential barrier for electron ejection in the presence of a SP resonance. More precisely, the minimum energies needed for an electron to be ejected after absorption of one photon $(\hbar \omega=4.15 \mathrm{eV})$ or after absorption of two photons $(\hbar \omega=2.43 \mathrm{eV})$ differ significantly. This suggests that the characteristics of the electron ejection mechanism itself are modified by the SP resonance. It has to be emphasized that the threshold shift has been unambiguously proven because our experiment enables us to measure directly the work function (out of $S P$ resonances) via continuous tuning of the laser wavelength.

It is worth noting that a shift in the Fröhlich frequencies of small alkali clusters has been observed and was interpreted as due to the spill-out of electron density at the surface when the SPs are excited. A comprehensive description of this effect and whether it corresponds 


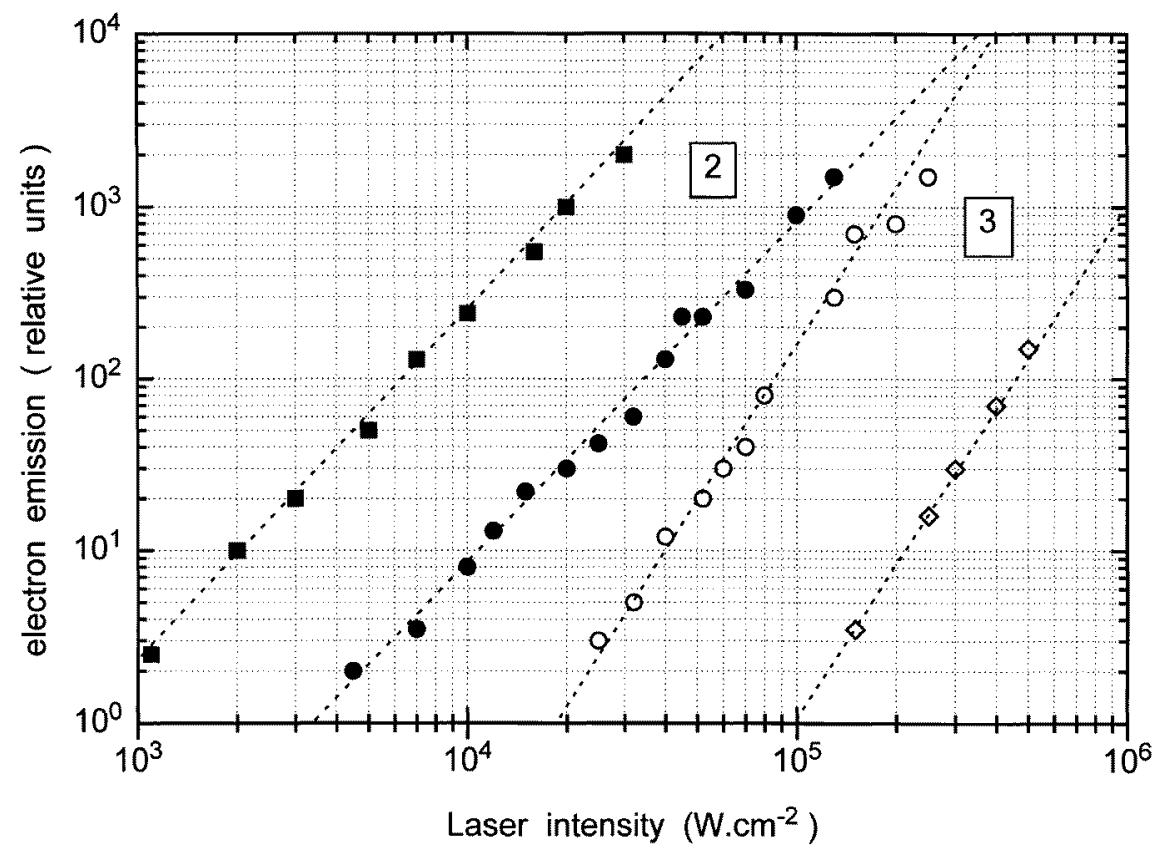

Figure 6. Lux-ampere characteristics of the electron signal measured across the third SP resonance around $500 \mathrm{~nm}$. The slopes corresponding to the emission process are shown to change from 2 to 3 (shown by the broken straight lines) between $480 \mathrm{~nm}(\bigcirc)$ and $510 \mathrm{~nm}(\bigcirc)$. Also shown are the data obtained at $370 \mathrm{~nm}(\boldsymbol{\square})$ and $550 \mathrm{~nm}(\diamond)$.

to a blue or a red shift for Ag clusters can be found in the literature (Kreibig and Vollmer 1995). It may therefore be thought that modifications of the electron characteristics at the surface can indeed be found in different experimental properties such as the Fröhlich frequencies or the work function. More theoretical work is needed in order to predict quantitatively how $W_{s}$ is modified. The shift of the two-photon threshold observed here may correspond effectively to such an effect. In that case, the shift should depend on the amplitude of the resonance if the resonance is not saturated. This could explain why such a shift is observed for the third SP resonance near $510 \mathrm{~nm}$, but not for the second or the first at 375 or $335 \mathrm{~nm}$. A simple way to check this effect would be to observe the variation in this shift as a function of the amplitude of the SP resonance. This implies, in other words, control of the shape and density of the different granular populations. Work is now in progress to achieve such a conclusive experiment.

\section{Acknowledgments}

We thank F Sabary and H Monard for the preparation of the silver film, and D Porterat for technical assistance at the early stages of the experiment.

\section{References}

Anisimov S I, Benderskii V A and Farkas G 1978 Sov. Phys.-Usp. 20467

Ashcroft N W and Mermin N D 1987 Solid State Physics (Philadelphia, PA: Holt, Rinchart and Winston)

Bechtel J H, Lee Smith W and Bloembergen N 1977 Phys. Rev. B 154557 
Bigot J Y, Merle J C, Cregut O and Daunois A 1995 Phys. Rev. Lett. 754702

Bohren C F and Huffman D R 1983 Absorption and Scattering of Light by Small Particles (New York: Wiley) p 326

Cardona M and Ley L 1978 Photoemission in Solids (Top Appl. Phys. 26) (Berlin: Springer) p 20

Dudek J C and Sabary F 1990 Vacuum 411469

Estabrook K and Kruer W L 1978 Phys. Rev. Lett. 4042

Fann W S, Storz R, Tom H W K and Bokor J 1992 Phys. Rev. B 4613592

Forstman F and Gerhardts R R 1986 Metal Optics near the Plasma Frequency (Berlin: Springer)

Fowler R H 1931 Phys. Rev. 3845

Kreibig U and Vollmer M 1995 Optical Properties of Metal Clusters (Berlin: Springer) and references therein

Kretschmann E 1971 Z. Phys. 241313

Hoheisel W, Jungmann K, Vollmer M, Weidenauer R and Träger F 1988 Phys. Rev. Lett. 601649

Logothetis E M and Hartman P L 1969 Phys. Rev. 187460

Lundqvist S and March N H 1983 Theory of the Inhomogeneous Electron Gas ed N D Lang (New York: Plenum) ch 5

Macek Ch, Otto A and Steinmann W 1968 Phys. Status Solidi 51 K59

Otto A 1968 Z. Phys. 216398

Raether H 1988 Surface Plasmons on Smooth and Rough Surfaces and on Gratings (Berlin: Springer)

Rudolf H W and Steinmann W 1977 Phys. Lett. 61A 471

Sabary F and Dudek J C 1990 Vacuum 41476

Sabary F, Dudek J C and Bergeret H 1991 J. Appl. Phys. 701066

Skillman D C and Berry C R 1968 J. Chem. Phys. 483297

Steinmuller-Nethl D, Hopfel R A, Gornik E, Leitner A and Aussenegg F R 1992 Phys. Rev. Lett. 68389

Stuckless J T and Moskovits M 1989 Phys. Rev. B 409997

Tsang T, Rao T S and Fischer L 1990 Opt. Lett. 15866

Wood D M 1981 Phys. Lett. 46749

Yen R, Liu J and Bloembergen N 1980 Opt. Commun. 35277 